\title{
Gemcitabine-Induced Cardiotoxicity in Patients Receiving Adjuvant Chemotherapy for Pancreatic Cancer: A Case Series
}

\author{
Salma Alam ${ }^{a} \quad$ Chidi Illo ${ }^{a} \quad$ Yuk Ting Ma ${ }^{b}$ Pankaj Punia ${ }^{a}$ \\ ${ }^{a} Q u e e n$ Elizabeth Hospital, Birmingham, UK; ${ }^{b}$ School of Cancer Sciences, University of \\ Birmingham, Birmingham, UK
}

\section{Keywords}

Gemcitabine $\cdot$ Cardiotoxicity $\cdot$ Pancreatic cancer

\begin{abstract}
Gemcitabine is not considered a cardiotoxic agent generally; so far only very few case reports have been reported in the literature on different aspects of cardiac side effects. Here we report a case series of 3 patients who developed congestive cardiac failure, when treated with gemcitabine monotherapy in the adjuvant setting for pancreatic cancers. Adjuvant chemotherapy with gemcitabine has been the standard of care for pancreatic cancer patients after successful surgery since the results of the CONKO-001 and ESPAC3 study were published. Gemcitabine was administered on days 1,8 , and 15 of a 28 -day cycle at $1,000 \mathrm{mg} / \mathrm{m}^{2}$. All 3 patients developed symptoms suggestive of cardiac failure with a drop in ejection fraction on echocardiography, and responded to conservative treatment for heart failure after withdrawal of gemcitabine therapy. Early withdrawal of gemcitabine chemotherapy is recommended in addition to a need for studies required to evaluate the mechanism of cardiotoxicity. As per available literature, patients with diabetes and having received a total dose greater than $15,000 \mathrm{mg} / \mathrm{m}^{2}$ are generally at a higher risk and require close surveillance.
\end{abstract}


 Oncology}

\section{Introduction}

Antineoplastic therapies have evolved considerably over the past decade, resulting in an overall survival, and improvement in morbidity and mortality for various different tumour types. Gemcitabine is a commonly used chemotherapy agent in the treatment of various solid malignancies including pancreatic, ovarian, breast, bladder, and non-small cell lung cancers. It is a nucleoside analogue and a pyrimidine antimetabolite that inhibits deoxyribonucleic acid (DNA) synthesis by inhibition of DNA polymerase and ribonucleotide reductase [1] resulting in apoptosis [2].

Gemcitabine is a relatively well-tolerated chemotherapy; the commonly reported side effects include bone marrow suppression, liver and kidney problems, nausea, fever, rash, shortness of breath, and hair loss. Its use during pregnancy increases the risk of teratogenesis. Cardiotoxicity, especially cardiomyopathy, has not been reported during early phase trials of drug development [3]. Although gemcitabine-induced cardiomyopathy is extremely rare, we report here 3 cases of gemcitabine-induced cardiomyopathy from a single tertiary centre in the United Kingdom.

\section{Case Report 1}

A 62-year-old Caucasian man first presented in November 2015 with recurrent epigastric and back pain, diarrhoea, vomiting, jaundice, and weight loss. Co morbidities included rheumatoid arthritis, peptic ulcer, hypertension, and myocardial infarction (15 years previously). He was on bisoprolol, piriton, simvastatin, amitriptyline, omeprazole, and adcal. He smoked 5 cigarettes per day and had a moderate alcohol intake.

Following an initial work-up he underwent a Whipple procedure. Histological examination revealed pT3N0 pancreatic carcinoma. Adjuvant chemotherapy with IV gemcitabine $\left(1,000 \mathrm{mg} / \mathrm{m}^{2}\right.$ to the nearest $38 \mathrm{mg}$ as $2,100 \mathrm{mg}$ given on day 1,8 , and 15 on a 28-day cycle for 6 cycles) was recommended and commenced to reduce recurrence following a case discussion at the multi-disciplinary team meeting.

Following the 4th cycle of chemotherapy he developed persistent peripheral oedema and myelosuppression with haemoglobin of 74 . He was given 2 units of blood transfusion, and echocardiography at this point showed normal heart and valvular function. He was treated with furosemide and given the option of dose reduction or stopping treatment due to cumulative toxicity. The patient wanted to continue treatment and therefore received a $25 \%$ reduced dose at cycle 5 . However on routine clinical follow-up, after cycle 5 for toxicity assessment, the peripheral oedema had worsened. Clinical examination revealed right-sided basal crepitation but normal JVP, his serum electrolytes were normal but haemoglobin was down to 73. The patient was adamant to continue and complete the treatment. It was therefore decided to delay treatment until symptoms improved. Despite a further 2 units of blood given as a day case and a higher dose of furosemide, oedema and shortness of breath got worse at home and he was admitted as an inpatient. His renal function began to deteriorate. A repeat echocardiogram showed an LVEF of $40 \%$ and moderate mitral valve regurgitation. Additionally, a high-resolution CT scan demonstrated multiple parenchymal infiltrates suggestive of possible pneumonitis with fluid overload. Nephrologists, cardiologists, and pulmonologists were involved in patient management. 
A decision was made to stop chemotherapy. He was treated with steroids, antibiotics with PCP cover, and diuretics, leading to symptomatic improvement and was discharged home.

The patient needed to be readmitted to hospital after a couple of weeks with worsening shortness of breath and pedal oedema. He was found to have pulmonary oedema and persistent deterioration in renal function requiring critical care review in the intensive therapy unit for respiratory support and haemofiltration. After a full renal screen by the renal medicine team, the patient's acute kidney injury was attributed to gemcitabine. A repeat highresolution CT scan demonstrated a progression of possible drug-induced pneumonitis. He continued to receive steroids, diuretics, and antibiotics. He improved after a couple of weeks and was discharged. The Cardiology Team however attributed the patient's heart failure to ischaemic heart disease despite the likelihood of gemcitabine-induced cardiotoxicity. However, a repeat echocardiogram 3 weeks later showed an improvement in cardiac function; LVEF 50\% and no valvular dysfunction were noted. He has not been readmitted since and is being followed up in the outpatient clinic by the oncology, nephrology, cardiology and respiratory medicine teams.

Following completion of adjuvant chemotherapy, albeit treatment ceased early due to toxicity, post-treatment imaging did not demonstrate disease recurrence. His care was therefore handed back to the surgical colleagues for further follow-up.

\section{Case Report 2}

A 63-year-old Caucasian man initially presented with painless jaundice against the background of no significant past medical history. He underwent a Whipple procedure for carcinoma of the head of the pancreas. Histology showed a pT3 tumour with the involvement of 8 lymph nodes. He commenced on adjuvant gemcitabine $\left(1,000 \mathrm{mg} / \mathrm{m}^{2}\right.$ to the nearest $38 \mathrm{mg}$ as 2,100 mg given on day 1, 8, 15 on a 28-day cycle, planned for 6 cycles).

Just prior to his 3rd cycle, he gradually developed bilateral pedal oedema, with no other signs or symptoms. He was started on furosemide and chemotherapy continued. During his 4th cycle he received 2 units of blood. At the start of his 5th cycle he developed occasional shortness of breath, but by day 15 he was found to be anaemic and thrombocytopenic with acute kidney failure. Investigations ruled out urinary obstruction. He was treated with intravenous fluids and blood products. Patient was discharged home after renal team review. After a week at home, peripheral oedema and breathlessness increased and he was readmitted to hospital. Chest X-ray showed a mixed picture of fluid overload and right upper lobe consolidation. An echocardiogram demonstrated impaired systolic function with LVEF of $38 \%$, mildly dilated left atrium, and mild mitral and tricuspid regurgitation. The patient was admitted to the coronary care unit by the cardiology team and was treated with antibiotics and diuretics, initially intravenously and later orally as he improved. It was decided to terminate adjuvant chemotherapy at the 5 th cycle.

The CT scan at this stage was clear and the patient was placed on surveillance and continued outpatient follow-up by the cardiology and nephrology teams. A further cardiac MRI arranged by the cardiology team 2 months later demonstrated stable cardiac function, with an improvement in the EF to 67\%, but demonstrated a suspicious retrocrural lymph node. To rule out recurrence a repeat CT staging scan was performed, additionally confirming that the retrocrural lymph nodes also showed multiple peritoneal metastases. 


\section{Case Reports in Oncology}

He was subsequently commenced on 2nd line FOLFOX (FOLFIRINOX modified due to renal and cardiac impairment) at a 75\% reduced dose; treatment was well tolerated with only grade 1 peripheral neuropathy affecting fingertips and fatigue. The CT scan after 6 cycles and on completion of the 12th cycle showed stable disease. A surveillance scan after 3 months demonstrated disease progression again with worsening peritoneal and omental disease. He was re-challenged with FOLFOX. He continued to tolerate chemotherapy with minimal toxicity. Unfortunately he quickly deteriorated after cycle 5 of re-challenge FOLFOX chemotherapy, with severe cachexia and anorexia. A repeat CT confirmed significant progressive disease in the peritoneum and omentum with an enlarged metastatic node in the right groin. He passed away a few days later in February 2017.

\section{Case Report 3}

A 72-year-old Caucasian female presented with painless jaundice in November 2014. Comorbidities included type II diabetes mellitus, hypertension, and hypercholesterolaemia. She was on insulin, ramipril, rosuvastatin and lansoprazole. She was an ex-smoker but had no history of alcohol consumption.

Imaging showed pancreatic abnormality and a right-sided lung mass. Biopsy confirmed 2 separate primaries; she underwent right-sided lobectomy and Whipple procedure. Histology showed a lung adenocarcinoma (T2N0R0) and a poorly differentiated ductal adenocarcinoma of the head of the pancreas with lymph node, perineural, and vascular invasion (pT3pN1).

She was started on adjuvant gemcitabine, planned for 6 cycles. $75 \%$ of the standard dose $\left(1,000 \mathrm{mg} / \mathrm{m}^{2}\right)$ was given at the 1 st cycle due to ongoing weakness after surgery, but then it was increased to $100 \%$ from 2 nd cycle as the 1 st cycle was well tolerated.

Following the 2nd cycle, she developed peripheral oedema, cough, and shortness of breath, which were treated with furosemide and antibiotics. On completion of the 3rd cycle, shortness of breath and peripheral oedema worsened, clinically suggestive of congestive cardiac failure. She was admitted for investigations. CTPA showed subtle ground-glass appearance and an echocardiogram showed LVEF $60-65 \%$ with moderate aortic stenosis but no LVH. A diagnosis of chemotherapy-induced pneumonitis with underlying undiagnosed COPD was made, although clinical examination also favoured cardiac failure with somewhat preserved LVEF. Good left ventricular function on the echocardiogram may be misleading in this case as the patient had already been on diuretics for a couple of week at the time of the echocardiogram. She was treated with steroids, antibiotics, and diuretics, and was started on salbutamol inhalers. It was decided to stop further gemcitabine treatment due to significant toxicity; imaging showed stable disease, and the patient was placed under surveillance. Shortness of breath gradually improved over the next months.

She had confirmed disease progression after 6 months of surveillance, with local recurrence, omental nodules, and liver lesions. She was commenced on 2 nd line FOLFOX. The 1st cycle of chemotherapy was not well tolerated with severe diarrhoea and nausea, leading to a dose reduction for 2 nd cycle to $80 \%$. She suffered similar toxicities after the 2 nd cycle, including grade 3 mucositis. Following this, she was not prepared to continue with chemotherapy and opted for best supportive care. 
 Oncology}

\section{Discussion}

Cardiotoxicity is a well-known side effect of various antineoplastic agents like anthracyclines and 5-fluorouracil chemotherapy agents. Many newer biologic therapies are also considered potentially cardiotoxic, e.g. trastuzumab and rituximab, but cardiotoxicity is not a commonly reported side effect of gemcitabine monotherapy [4]. It should be used with caution in patients with liver, kidney, or cardiovascular disorders. In rare cases it may cause posterior reversible encephalopathy syndrome, capillary leak syndrome, severe lung conditions like pulmonary oedema, pneumonia, and adult respiratory distress syndrome $[5,6]$.

There have been case reports in the literature of acute myocardial infarction, arrhythmias like SVT and pericarditis following the use of gemcitabine but there is a paucity of data on gemcitabine-induced cardiomyopathy. Shrum et al. [7] published a retrospective analysis of the case notes of 156 patients to further investigate and assess clinical features which may predispose patients to developing new-onset gemcitabine-induced cardiomyopathy. All of the patients had received gemcitabine for various cancers in 1st or subsequent lines of treatment, 51 patients in ovarian cancer, and 105 patients with breast, lung, pancreas, and bladder cancer. Patients with new-onset congestive heart failure were compared with patients without new-onset congestive heart failure with the use of Wilcoxon rank-sum test and the Fisher exact test. $4.5 \%$ of patients developed new-onset congestive cardiac failure, which is more than previously reported $(0.76 \%$ in the early phase trials) [8]. This patient cohort did not differ much in baseline characteristics such as age, weight, parity, body mass index, prior therapy with Adriamycin or smoking history. Interestingly, a comorbidity of diabetes mellitus and coronary artery disease was more common in addition to having received a total dose of $>17,000 \mathrm{mg} / \mathrm{m}^{2}$ in patients who developed new-onset congestive heart failure with gemcitabine. The authors therefore recommended additional follow-up for surveillance and screening of cardiac toxicity for all patients who have received $>15,000 \mathrm{mg} / \mathrm{m}^{2}$ of gemcitabine.

The French PharmacoVigilance Database (FPVD) also identified gemcitabine as one of the drugs [9] which causes drug-induced cardiomyopathy following a retrospective analysis of case reports over 16 years between 1 January 1990 and 30 June 2007; anthracyclines were used as control. A total of 258,729 adverse drug reactions were recorded; 47 were defined as dilated cardiomyopathy. A significant reporting odds ratio was found with cytotoxic agents such as epirubicin, mitoxantrone, cyclophosphamide, gemcitabine, and fluorouracil. Despite limitations of the retrospective analysis, the above study highlighted that agents like gemcitabine deemed safe from cardiotoxicity could represent a new pharmacovigilance signal and could contribute to establish further prospective studies in order to confirm such signals.

Khan et al. [10] published a comprehensive literature review and case report of gemcitabine-induced cardiomyopathy as a late or chronic side effect and other acute cardiac events. Acute cardiac events were described as myocardial infarct, angina, and/or arrhythmias. The exact mechanism of cardiac damage causing cardiomyopathy remains unknown.

Recently Mohebali et al. [11] described a case report of an elderly patient who had received multiple lines of chemotherapy, had stable cardiac function prior to commencing rituximab, gemcitabine, and oxaliplatin (R-Gem-Ox) for relapsed diffuse large B cell lymphoma and was admitted with symptoms of cardiac failure. The authors hypothesized that the patient was suffering from a multi-hit mechanism of cardiac muscle damage by potentially non-cardiotoxic agents resulting ultimately in cardiomyopathy. 
In the current age of the availability of electronic information and social media, eHealthMe, a personalized health information app, has been available to patients to help them look up potential side effects reported by patients of the same gender, age, etc. Up to 1 May 2017, 13,900 people, reported to have experienced side effects when taking gemcitabine. Among them, 30 people $(0.22 \%)$ had cardiomyopathy, especially male patients, $60+$ years old (incidence $47.37 \%$ ) who had been taking the drug for 1-6 months $(66.67 \%)$, were on frusemide (23.33\%), and had pancreatic carcinoma (13.33\%).

\section{Conclusion}

All 3 patients in our case series with reported cardiac toxicity improved following withdrawal of gemcitabine treatment. Stopping gemcitabine treatment in cases of potential cardiotoxicity should be the 1st step in the management of these patients. An echocardiogram prior to starting treatment in patients more than 50 years of age with diabetes mellitus and previous coronary artery disease may also be warranted. Further research to look into the mechanism of gemcitabine cardiotoxicity is required. Additionally, as suggested in the literature, patients who have received a dose higher than $15,000 \mathrm{mg} / \mathrm{m}^{2}$ should be screened for cardiotoxicity whilst continuing surveillance.

\section{Statement of Ethics}

Patient consent was obtained for publication.

\section{Disclosure Statement}

We have no conflict of interest to disclose.

\section{Funding Sources}

No funding was required for this case series.

\section{References}

1 Plunkett W, Huang P, Xu YZ, Heinemann V, Grunewald R, Gandhi V. Gemcitabine: metabolism, mechanisms of action, and self-potentiation. Semin Oncol. 1995 Aug;22(4 Suppl 11):3-10.

2 Aapro MS, Martin C, Hatty S. Gemcitabine - a safety review. Anticancer Drugs. 1998 Mar;9(3):191-201.

3 Carmichael J, Fink U, Russell RC, Spittle MF, Harris AL, Spiessi G et al. Phase II study of gemcitabine in patients with advanced pancreatic cancer. Br J Cancer. 1996 Jan;73(1):101-5.

4 Mattiucci GC, Ippolito E, D’Agostino GR, Alfieri S, Antinori A, Crucitti A et al. Long-term analysis of gemcitabine-based chemoradiation after surgical resection for pancreatic adenocarcinoma. Ann Surg Oncol. 2013 Feb;20(2):423-9.

5 Gemcitabine "UK label." UK Electronic Medicines Compendium. 5 June 2014 [cited 2017 May 6].

$6 \quad$ "Gemcitabine." Macmillan Cancer Support. 2017 [cited 2017 May 6].

7 Shrum KJ, Gill SE, Thompson LK, Blackhurst DW, Puls LE. New-onset congestive heart failure with gemcitabine in ovarian and other solid cancers. Am J Clin Oncol. 2014 Aug;37(4):364-8.

8 Storniolo AM, Allerheiligen SR, Pearce HL. Preclinical, pharmacologic, and phase I studies of gemcitabine. Semin Oncol. 1997 Apr;24(2 Suppl 7):S7-2. 
Alam et al.: Gemcitabine-Induced Cardiotoxicity in Patients Receiving Adjuvant Chemotherapy for Pancreatic Cancer: A Case Series

9 Montastruc G, Favreliere S, Sommet A, Pathak A, Lapeyre-Mestre M, Perault-Pochat MC et al.; French Association of Regional PharmacoVigilance Centres. Drugs and dilated cardiomyopathies: a case/noncase study in the French PharmacoVigilance Database. Br J Clin Pharmacol. 2010 Mar;69(3):287-94.

10 Khan MF, Gottesman S, Boyella R, Juneman E. Gemcitabine-induced cardiomyopathy: a case report and review of the literature. J Med Case Reports. 2014 Jun;8(1):220.

11 Mohebali D, Matos J, Chang JD: Gemcitabine induced cardiomyopathy: a case of multiple hit cardiotoxicity. ESC Heart Fail. 2017 Feb;4(1):71-4. 